CASE REPORT

\title{
Giant Mesenteric Lipoma Causing Small Bowel Volvulus: A Case Report
}

Yisihak Suga $^{1^{*}}$, EsakAbdi $^{1}$, Mahteme Bekele $^{1}$

\author{
OPEN ACCESS \\ Citation: Yisihak Suga, EsakAbdi, \\ Mahteme Bekele. Giant Mesenteric \\ Lipoma Causing Small Bowel Volvulus: \\ Case Report. Ethiop J Health \\ Sci.2017;29(4):529. doi:http://dx.doi.org/ \\ 10.4314/ejhs.v29i4.15 \\ Received: March 20, 2019 \\ Accepted: May 15, 2019 \\ Published: July 1, 2019 \\ Copyright: (c) 2019 Yisihak Suga, et al. \\ This is an open access article distributed \\ under the terms of the Creative Commons \\ Attribution License, which permits \\ unrestricted use, distribution, and \\ reproduction in any medium, provided the \\ original author and source are credited. \\ Funding: Nil \\ Competing Interests: The authors \\ declare that this manuscript was approved \\ by all authors in its form and that no \\ competing interest exists. \\ Affiliation and Correspondence: \\ ${ }^{1}$ Department of surgery, Saint \\ Paul's Hospital Millennium Medical \\ College (SPHMMC), Addis Ababa, \\ Ethiopia \\ *Email: yisihaksuga12@gmail.com
}

ABSTRACT

BACKGROUND: A lipoma of the small bowel mesentery is a rare clinical entity. It rarely causes intestinal obstruction mainly due to volvulus.

CASE: We report a case of a 25 years old male who presented with acute exacerbation of abdominal pain, nausea and vomiting. Plain abdominal $x$-ray showed complete small bowel obstruction. At laparotomy, there was small bowel volvulus with a big yellow mass as an axis. Enbloc resection and end-to-end ileal anastomosis was done.

CONCLUSION; Mesenteric lipoma are rare. They should be considered in cases of long standing abdominal pain.

KEYWORDS: lipoma, mesentery, Intestinal obstruction

\section{INTRODUCTION}

Lipoma is the most common benign tumor of soft tissues in the human body. They were first described in 1757, by Bauer (1). They occur in elderly people. The majority are subcutaneous in location. They may develop virtually in all organs through the body.

One of the rare sites for lipoma to occur is small bowel mesentery, which is called mesenteric lipoma $(1,3)$. Mesenteric lipoma is usually diagnosed accidentally, and most are asymptomatic as it usually allows the passage of intestinal contents. If patients develop symptoms, the common ones are abdominal swelling and abdominal pain $(2,4,5)$. Rarely, the presentation could be with complications of the mass like small bowel obstruction with volvulus.

In this article, we present a case of small bowel obstruction secondary to volvulus resulted from mesenteric lipoma. The clinical presentation and pathology description are discussed with review of literature.

\section{THE CASE}

A 25 years old male Ethiopian patient presented with worsening of intermittent abdominal pain of three days duration. Associated with this, he also had frequent vomiting of bilious matter, progressive abdominal distention and later failure to pass feces and flatus. He had visited a health center several time for his long standing abdominal cramp, but he was only given anti parasite and anti-pain. 
Physical examination revealed tachycardia of 100 beat/minute. Abdominal examination revealed a distended abdomen with visible peristalsis, hyper tympanic on percussion and scanty stool in the rectum during digital rectal examination. laboratory investigation results were Unremarkable

Plain abdominal x-ray showed centrally located, multiple air fluid level with stepladder appearance.

With an assessment of small bowel obstruction secondary to primary volvulus, the patient was resuscitated with fluid, NG tube inserted and
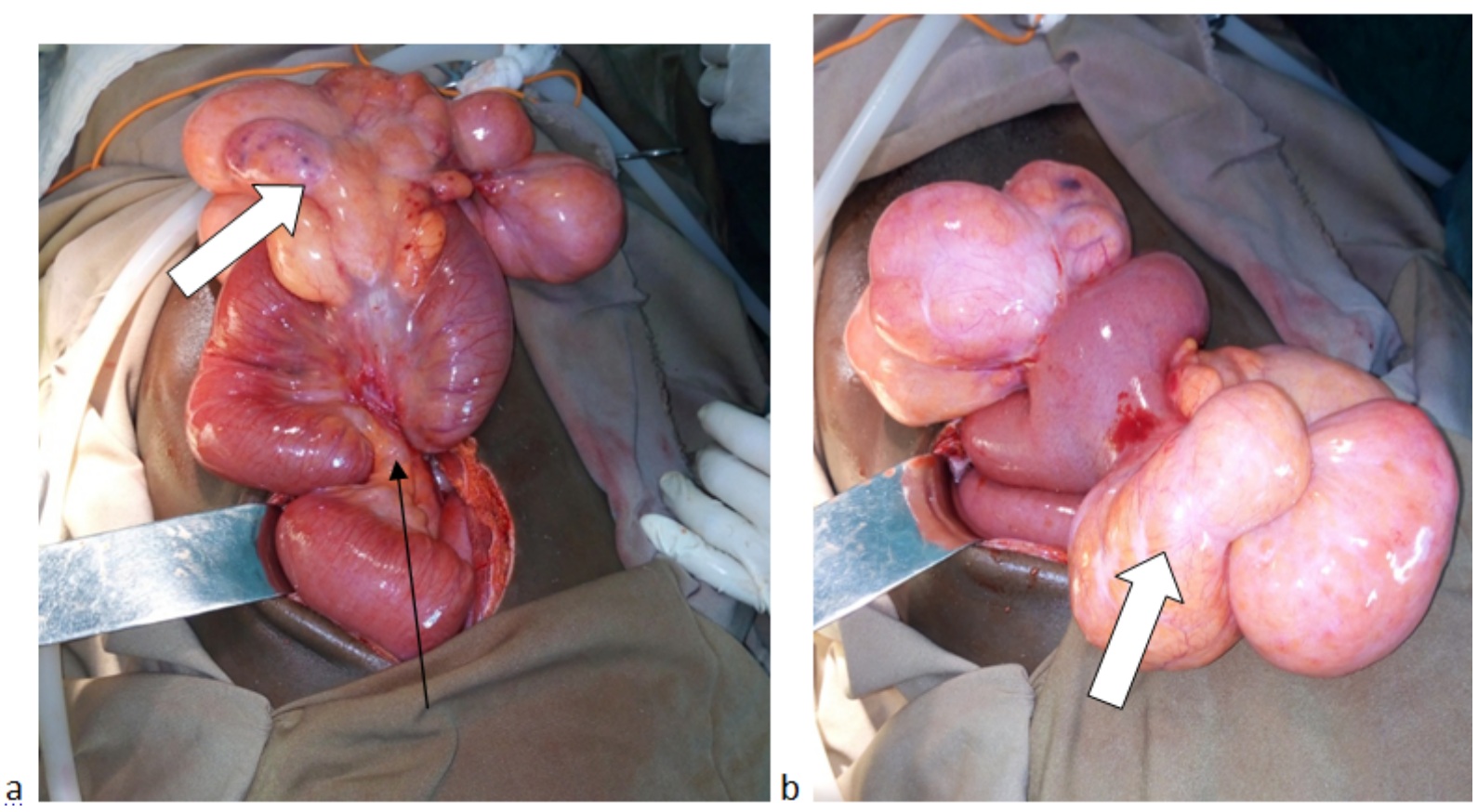

Figure 1: a) volvulated small bowel (black arrow) with a mass as an axis (white arrow); b) lobulated big yellow mass (white arrow head) on the mesenteric side of the bowel

For this, the reactive fluid was sucked out, the volvulus was de-rotated and the mass was resected en-block with segment of the bowel (Figure 2). End-to-end ileo-ileal anastomosis was done. The resected specimen was subjected to histopathology examination

The histology examination showed a well circumscribed tissue composed of lobules of mature adipose tissue aggregates of mononuclear catheterized and produced adequate urine. Informed consent was obtained for explorative laparotomy. He was explored through a midline vertical incision. There was minimal reactive fluid, a distended small bowel which volvulated 720 degree anti-clockwise over a big mass which is $80 \mathrm{~cm}$ from ileo-cecal valve. The mass was big $(15 \times 10 \mathrm{~cm})$, yellowish in color, multi lobulated. Arises from small bowel mesentery involving its wall partially but the bowel lumen was patent and viable with no perforation. There were few mesenteric lymphadenopathies (Figure 1). 


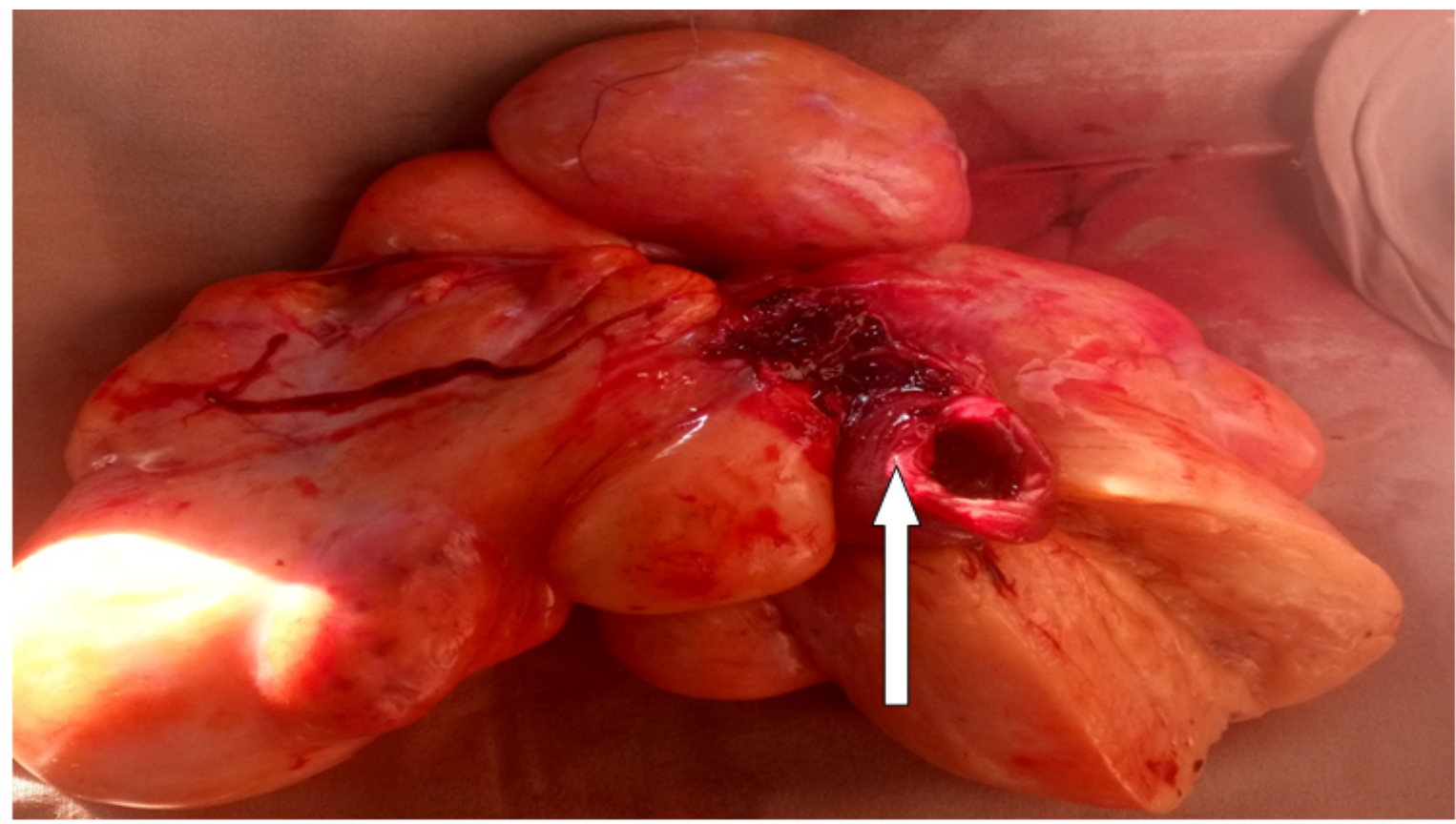

Figure 2: Resected specimen gross appearance with segment of small bowel (arrow head) and mesenteric mass

A
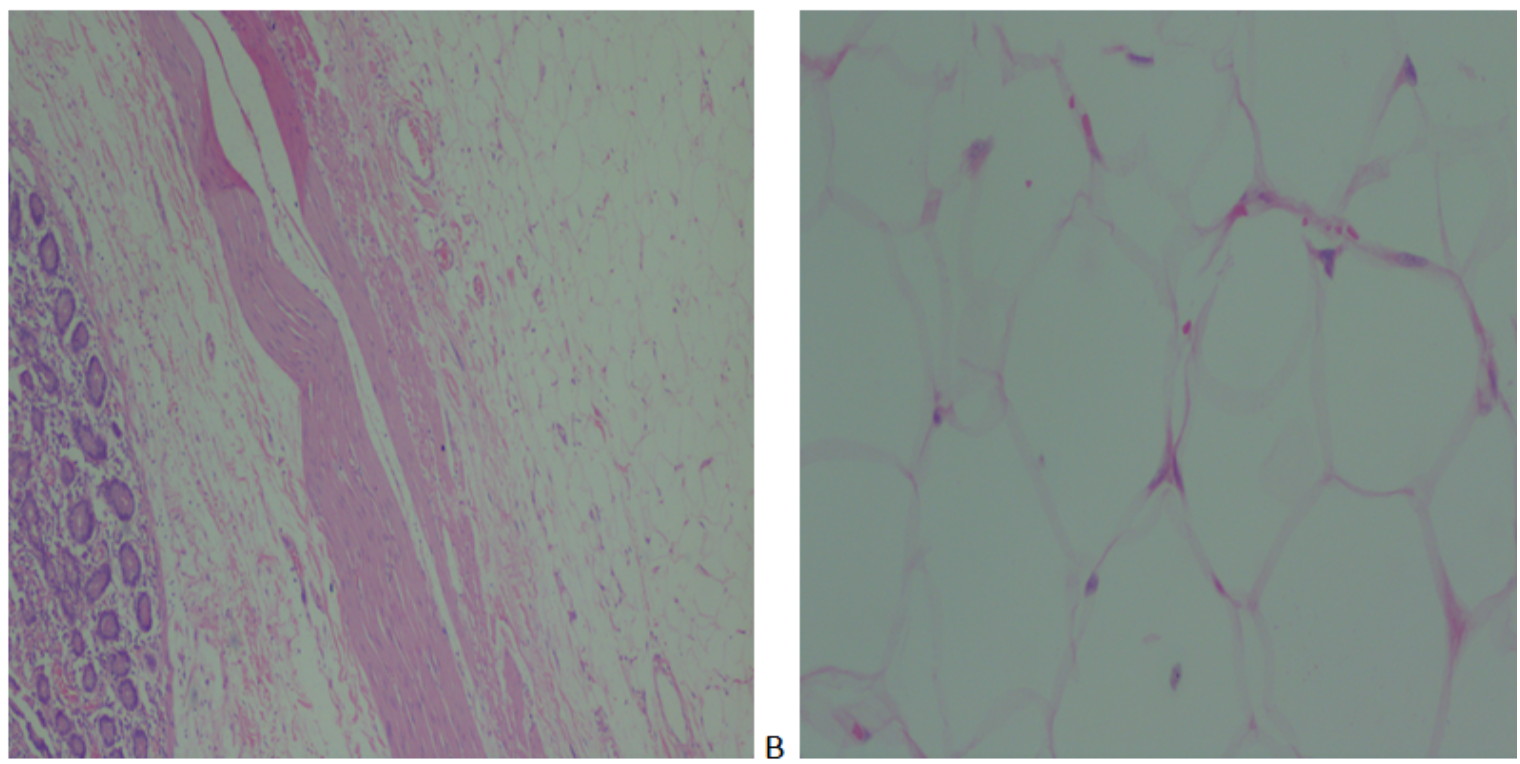

Figure 3 (A, B: Histopathology result: shows microscopic appearance of adipose tissue Biopsy code; SP18

\section{DISCUSSION}

Mesenteric lipomas are rare clinical entities, with less than 50 cases described in English language literature till $2014(2,3,4)$. To the best of our knowledge, this is the second report of mesenteric lipoma from Africa $(1,3)$. This shows the rarity of this clinical entity.

Mesenteric lipoma is commoner in adults aged between 40 and 60 years and rarely occurs in children of less than 10 years (4). It is said to have

DOI: http://dx.doi.org/10.4314/ejhs.v29i4.15 
slight female preponderance. The factors that predispose to lipoma are obesity, diabetes mellitus, hypercholesterolemia, familial tendency, trauma, radiationtherapy and chromosomal translocation $(2,5)$. Our patient was a 25 years old male with no identifiable etiologic factors.

Small intestinal mesenteric lipomas mostly occur on the ileum $(1,2,5)$. They either occur in the root of the mesentery or at the luminal edge of the mesentery. This was also the case in our patient.

These lipomata are usually asymptomatic because most allow passage of small bowel as the lipomas are soft and mobile masses which do not infiltrate into surrounding structures (34).

For symptomatic patients it is related to the pressure effects of big lipoma. The common symptoms are vague abdominal pain, distension, and anorexia and weight loss (5). Rarely, patients present with signs and symptoms of intestinal obstruction, and this is usually due to small bowel volvulus or intussusception caused by the lipoma. The case presented with long standing abdominal pain and signs and symptoms of acute intestinal obstruction.

Mesenteric lipomas are diagnosed accidentally after laparoscopy or laparatomy as most have no symptoms. MRI and Computed Tomography (CT) is also said to be useful in diagnosis. Ultrasound shows a well defined homogenous echogenic mass, and so can distinguish it from a mesenteric cyst. Computed Tomography (CT) shows homogenous tumor of adipose tissue. Also gives information about effect on the small bowel, whether there is evidence of ischemia and may demonstrate the typical whirl like pattern of a volvulus $(1,4)$. Unfortunately, our patient didn't have the opportunity to have CT scan as he presented acutely. We took plain abdominal x-ray which showed complete small bowel obstruction which is one of the complications of the clinical entity.

Treatment of large or symptomatic mesenteric lipomata involves surgical excision of the lipoma with resection of the adjacent small bowel and end-to-end anastomosis (3,4). However, enucleation of tumor from the leaves of mesentery has been reported. Laparoscopic excision of mesenteric lipoma has also been reported.
Liposarcomas have also been described in the literature, rarely arising from the small bowel mesentery. Even if mesenteric lipomata are rare, they should be considered in cases of long standing abdominal pain, especially in resource limited areas where most of these patients will be seen first by non-physicians. The treatment involves laparatomy with enbloc resection the segment of the bowel.

\section{REFERENCES}

1. MajAmitAgrawal, Col KJ Singh. Symptomatic intestinal lipomas: our experience. MJAFI 2011;67:374-376.

2. ChristelleTayeh, SirinMneimneh, Rayan ElMasri, Nabil Daoud, Mariam Rajab Giant mesenteric lipoma: A case report and a review of the literature J PedSurg Case Reports 3 (2015) 166e170

3. ChristelleTayeh, SirinMneimneh, Rayan ElMasri, Nabil Daoud. Mariam Rajab, Giant Mesenteric Lipoma: A Case Report and a Review of the Literature Article in Journal of Pediatric Surgery Case Reports · February 2015

4. Yashpal, M Bansal, AKudva Small Bowel Intussusception in an Adult due to Lipoma: aRare Cause of Obstruction. Case report and LiteratureReview The Internet Journal of SurgeryVolume 25 Number 1

5. DG Watt1, P Sanjay, SV Walsh2, JA Young, A Alijani Mesenteric Lipoma Causing Small Bowel Perforation: A Case Report \& Review of Literature Article in Scottish medical journal· November 2012 